\title{
Novel liquid-liquid transition in the liquid Se-GeSe system
}

\author{
Yoshimi Tsuchiya \\ Department of Physics, Faculty of Science, Niigata University, Ikarashi 2-8050, Nishi-ku, Niigata 950-2181, Japan
}

\begin{abstract}
The sound velocity in the liquid Se-SeGe system under the saturated vapor pressure was measured at every 1 degree. A minimum in the sound velocity as a function of temperature has been observed for alloys with 12.5 to 45 at.\% Ge, which suggests that the crossover liquid-liquid transition occurs in the liquid around $\mathrm{GeSe}_{2}$. In addition to the minimum, it has been found for alloys with 15 to 27.5 at.\% Ge that the sound velocity decreases rather stepwise. The reproducibility has been checked for different samples with the same composition and for different ultrasonic transducers. The decrease occurs in a very narrow temperature interval, a few degrees, and shows hysteresis depending on thermal treatments. The position of this temperature region lowers with increasing Ge concentration, and appears to merge into a minimum in the sound velocity around $\mathrm{GeSe}_{2}$. Novel structural changes, being the first order in nature, occur slightly below the crossover transition.
\end{abstract}

\section{Introduction}

The liquid Ge-Te around the eutectic composition ( 15 at/\% Ge) shows unique thermodynamic properties [1]. The molar volume shows the water-like density anomaly just above the eutectic temperature [2]. The amount of volume contraction is as large as $-7 \%$ in the temperature interval of $40 \mathrm{~K}$. This anomalous volume contraction is accompanied with a strongly peaked extremum in the specific heat, isothermal compressibility and thermal expansion coefficient as a function of temperature. It has been accepted that those unusual thermodynamic properties are indications of liquid-liquid crossover transitions. Although the specific heat of $\mathrm{Ge}_{15} \mathrm{Te}_{85}$ has a very sharp peak, the transition appears to proceed continuously.

The Ge-Se system is well-known for its excellent glass forming ability [3]. Therefore, a large number of experimental and theoretical works have been done for nearly half a century [4]. The preceding experiments on the molar volume [5] and sound velocity [6] suggest structural changes of a crossover type at high temperatures above $900^{\circ} \mathrm{C}$, however the maximum temperatures covered by these investigations were not high enough to definitely show the liquid-liquid crossover transition in liquid $\mathrm{GeSe}_{2}$. Neutron diffraction experiments at three temperatures have shown that structure changes over $300 \mathrm{~K}$ temperature intervals [4]. However, it has not been clarified yet whether or not the thermodynamic functions of the Ge-Te system have characteristic of liquid-liquid transition as observed in the liquid Ge-Te system. Evidences for the crossover transition in the liquid Ge-Se system seem to be still racking.

In this work, new sound velocity measurements are reported. It is shown that the structural change in liquid $\mathrm{GeSe}_{2}$ is of a crossover transition. In addition an unexpected step-like change in the sound velocity as a function of temperature has been found, which suggest that novel structural changes, being the first order in nature, occur in the Se rich side of liquid Ge-Se alloy slightly below the crossover transition.

\section{Experimental}

Sound velocity was measured using a PC controlled system consisting of LeCroy LT262 oscilloscope, Panametric 5077 pulsar and receiver, and Ohkura EC5500 digital temperature. The control program is a homemade program on Visual Basic [7]. With this system the sound velocity can be measured quasi-continuously. A specimen was

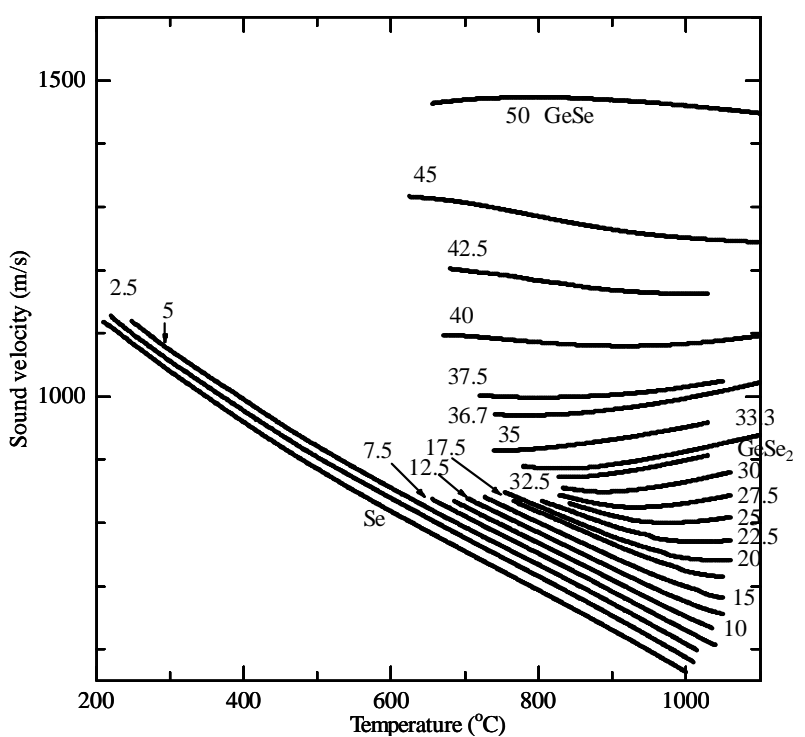

Fig. 1. Sound velocity in the liquid Se-SeGe alloy. Numbers are at.\% of Ge.

enclosed in a molten silica cell, which could be used up to $1100^{\circ} \mathrm{C}$ if the cell stands for the inner vapor pressure.

\section{Results and discussion}

Fig. 1 shows an overview of sound velocity as a function of temperature and its evolution with Ge concentration. The concentration of $\mathrm{Ge}$ in a sample is indicated by a number on a curve. A minimum indicating a liquid-liquid transition 


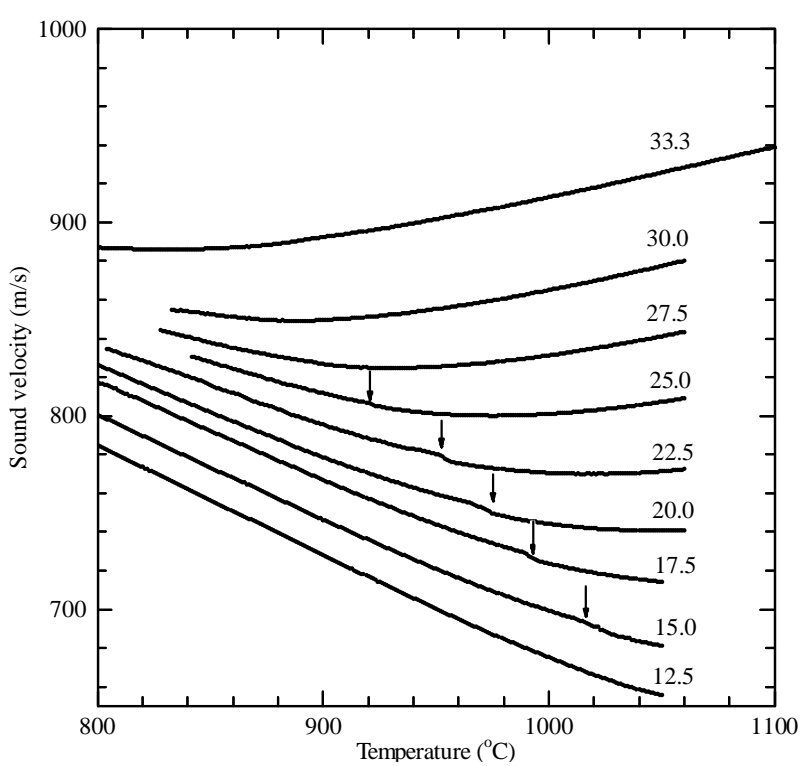

Fig. 2. Close-up of fig.1. Sound velocity in the liquid Ge-Se alloys with Ge concentration from 12.5 to 33.3 at.\% $\left(\mathrm{GeSe}_{2}\right)$. An arrow indicates the position of step-wise change, $T_{\mathrm{x}}$. Numbers are at.\% of Ge.

can be clearly seen for alloys around $\mathrm{GeSe}_{2}$ on this scale. The position of the minimum, referred to as the crossover transition temperature and denoted by $T^{*}$ hereafter, decreases with increasing Ge concentration up to around $\mathrm{GeSe}_{2}$ and increases with further increasing Ge concentration (see in Fig. 5). A close-up of Fig. 1 is shown in Fig. 2. In this figure, very small but definite step-wise change can be seen. The position of the step indicated by an arrow shifts to the lower temperature with increasing $\mathrm{Ge}$

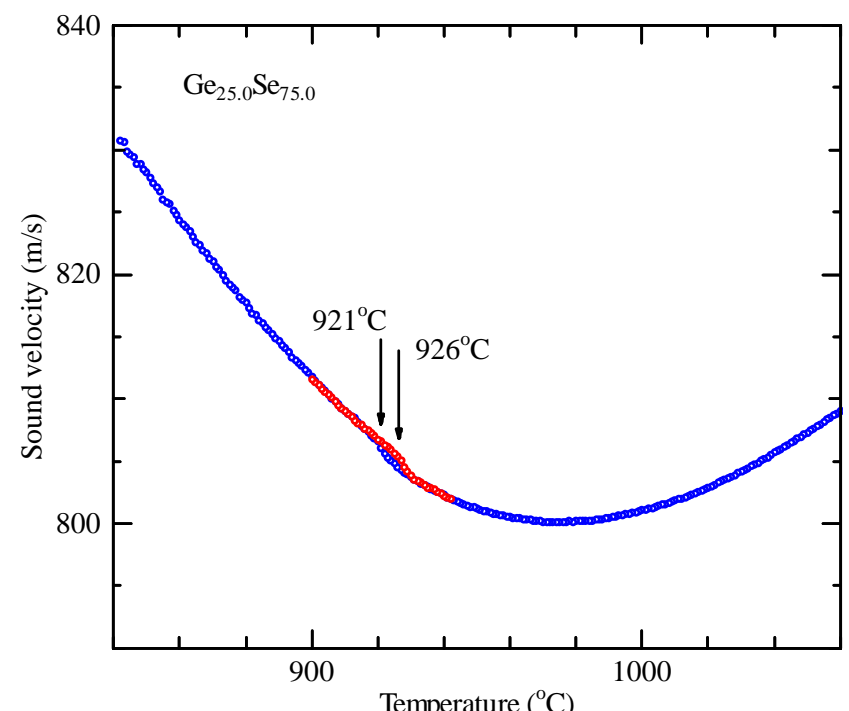

Fig. 3. Sound velocity in liquid $\mathrm{Ge}_{25} \mathrm{Se}_{75}$ measured in the cooling process (blue symbols) is compared with those measured in the heating process (red symbols). Slight hysteresis can be seen in the temperature interval between 921 and $926^{\circ} \mathrm{C}$ indicated by arrows.

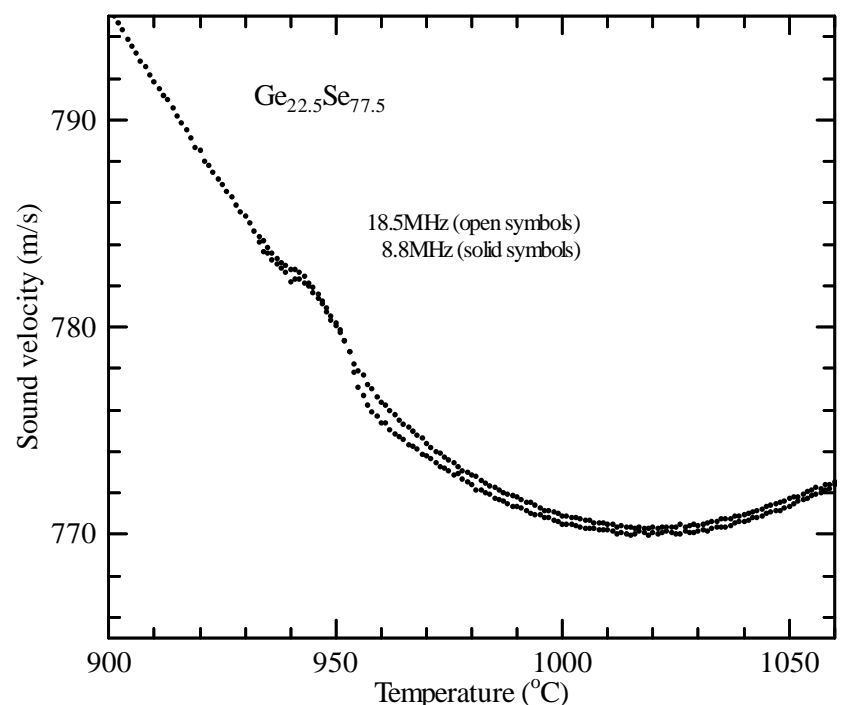

Fig. 4. Sound velocity in liquid $\mathrm{Ge}_{22.5} \mathrm{Se}_{77.5}$ measured with different frequencies. in the cooling process (blue symbols) is compared with those measured in the heating process (red sysmbols). Slight hysteresis can be seen in the temperature interval between 921 and $926^{\circ} \mathrm{C}\left(\mathrm{GeSe}_{2}\right)$. An arrow indicates the position of step-wise change, $T_{\mathrm{x}}$. Numbers are at.\% of Ge

concentration. The magnitude of step decreases as the $\mathrm{GeSe}_{2}$ is approached.

To investigate that such unexpected change is not artifact, the reproducibility was checked as in the following. 1) Measurements were made cyclically with cooling and heating processes and also the time for thermal equilibrium at each temperature was changed from 1 to 5 minutes. 2) Measurements were made for a few different samples with the same Ge concentration. 3) Ultrasonic transducer with different resonance frequency was also used if any frequency dependent existed. Two examples for such measurements are shown in Figs. 3 and 4.

Of quit interest is that the results for $\mathrm{Ge}_{25.0} \mathrm{Se}_{75.0}$ measured in the heating and cooling processes shows small hysteresis as plotted in Fig. 3. Fig. 4 compares the data measured with two different transducers with different resonance frequencies. Apart from small differences, $\sim 1$ $\mathrm{m} / \mathrm{s}$, in the magnitude at high temperatures, the results appear to be independent of the frequency of the ultrasound. Especially, no frequency dependence of the position of the step can be seen. The difference in the magnitude of the sound velocity at high temperatures could be brought about by differences in the signals of the sound wave caused by using different transducers, because the oscilloscope measures the time difference at their maxima of the selected oscillations. With those additional measurements, it is tentatively assumed that the step-wise change in the sound velocity is not artifact but indications of novel liquid-liquid transitions. The minimum position, $T^{*}$, and the position of the step-wise change, $T_{\mathrm{x}}$, are plotted in the phase diagram as shown in Fig. 5. $T^{*}$ takes a minimum at slightly Ge rich side of $\mathrm{GeSe}_{2}$ compound. 


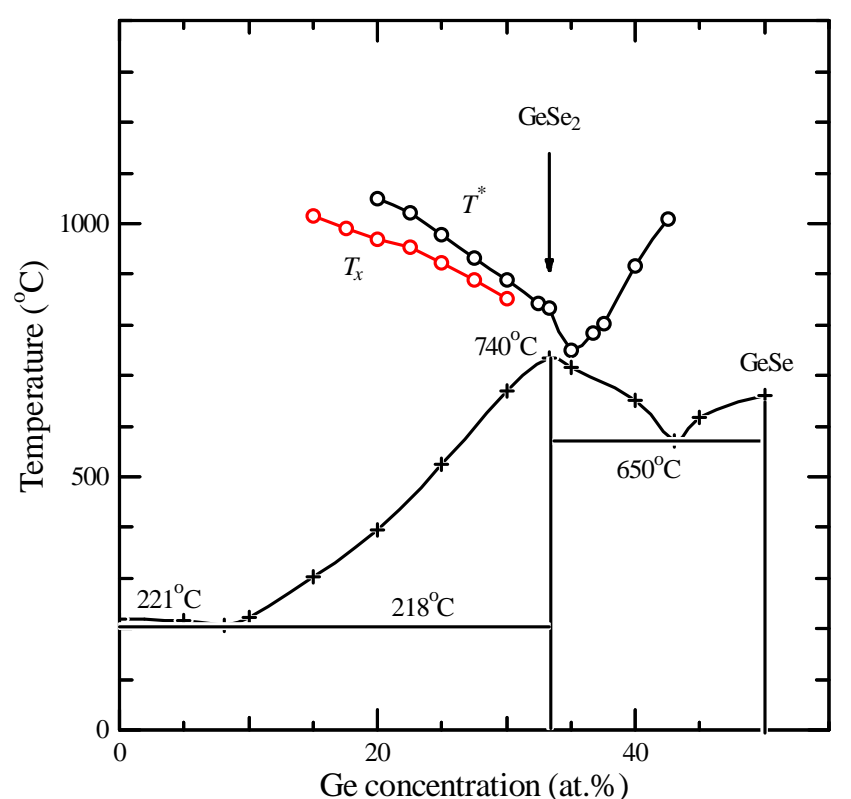

Fig. 5. Phase diagram of the Se-GeSe mixture. $T^{*}$ denotes the position of a minimum in the sound velocity and $T_{\mathrm{x}}$ the position of step-wise change.

As discussed in detail [9], the crossover liquid-liquid transition in mixture induces the concentration fluctuations. The relevant thermodynamic relation whether the concentration fluctuations are induced or not is given by

$$
-\frac{\delta C_{p}}{T^{*}}\left(\frac{\partial T^{*}}{\partial x}\right)^{2}
$$

where $\delta C_{p}$ is the specific heat associated with the crossover transition. The present results indicate that $\partial T^{*} / \partial x \approx 0$ occurs at Ge-rich side of $\mathrm{GeSe}_{2}$ and therefore the structural changes in liquid $\mathrm{GeSe}_{2}$ precede with inducing large concentration fluctuations. This is consistent with the fact that concentration fluctuations have been suggested in experiment showing the presence of considerable amount of Ge-Ge homopolar bonding in liquid $\mathrm{GeSe}_{2}$.

For the crossover transitions in liquid Te and Te-rich GeTe alloys, it seems now generally accepted that a spontaneous symmetry breaking mechanism called Peierls distortion play an essential role. In this case, structural changes are simplified as follows. At high temperatures an isotropic local structure similar to a sixfold coordinated simple cubic structure based on $p$ orbital bonding is realized. With lowering temperature, deformation to an anisotropic local structure with shorter and longer bonds takes place because of the energy gain called Peierls distortion. It is suggested recently that change in the vibrational entropy associated with change in the above mentioned bonding mechanism takes most part of entropy gain. On the other hand, for liquid $\mathrm{GeSe}_{2}$, first principle molecular dynamics simulations are mainly concentrated in explaining the origin of a first sharp diffraction peak and the overall features of the static structure. At low temperatures the structure factor has been correlated with formation of the $\mathrm{GeSe}_{4}$ tetrahedron. No theoretical investigation compared to the Peierls distortion mechanism seems to be presented for the stability of the $\mathrm{GeSe}_{4}$ tetrahedron.

\section{Summary}

Detailed sound velocity measurements revealed that liquidliquid cross-over transition occurs in Se rich Ge-Se mixtures. The crossover temperature $T^{*}$ takes a minimum at slightly Ge-rich side of $\mathrm{GeSe}_{2}$. The formal theory of crossover transition suggests that the crossover transition in liquid $\mathrm{GeSe}_{2}$ induces the concentration fluctuations in accord with experimental and theoretical structural investigations. In the sound velocity as a function of temperature a step-like change has been found for the first time, which suggests that novel liquid-liquid transition, being the first order in nature, occurs. However what actually happens in this system remains open to further investigations.

\section{References}

1. Y. Tsuchiya, J. Non-Cryst. Solids 212, 312 (2002)

2. Y.Tsuchiya, J. Phys. Soc. Jpn. 60, 227 (1991)

3. Sarrach et al., J Non-Cryst. Solids 22, 245 (1976)

4. P. S. Salmon, J Non-Cryst. Solids 353, 2959 (2007)

5. J. Ruska, H. Thurn, J. Non-Cryst. Solids 22, 227 (1976)

6. Y. Tsuchiya, J. Non-Cryst. Solids 122, 205 (1990)

7. Y. Tsuchiya, J. Phys. Condens. Matter 11, 593 (1999)

8. C. Massobrio, A. Pasquarello, R. Car, Phys. Rev. Lett. 80, 2342 (1998)

9. M. Micoulaut, R. Vuilleumier, C. Massobrio, Phys. Rev. B 79, 214205 (2009)

10. C. Otjacques, J. -Y. Raty, M.-V. Coulet, M. Jonson. H. Schober, C. Bichara, J. -P. Gaspard, Phys. Rev. Lett. 103, 245901 (2009) 